\title{
Glioblastoma stem cells and the importance of endolysosomes to keep them in the niches
}

\author{
Bernard Rogister ${ }^{1,2}$ \\ ${ }^{1}$ Laboratory of Nervous System Disorders and Therapy, GIGA-Neurosciences Research Center, University of Liège, Liège, Belgium; ${ }^{2}$ Department \\ of Neurology, CHU and University of Liège, Liège, Belgium \\ Correspondence to: Bernard Rogister, MD, PhD. GIGA-Neurosciences, Avenue Hippocrate, 15, 4000 Liège, Belgium. Email: Bernard.Rogister@ulg.ac.be. \\ Provenance: This is an invited Commentary commissioned by Section Editor Lichao Sun [State Key Laboratory of Molecular Oncology, National \\ Cancer Center (NCC)/Cancer Hospital, Chinese Academy of Medical Sciences (CAMS), Peking Union Medical College, Beijing, China]. \\ Comment on: Shingu T, Ho AL, Yuan L, et al. Qki deficiency maintains stemness of glioma stem cells in suboptimal environment by downregulating \\ endolysosomal degradation. Nat Genet 2017;49:75-86.
}

Submitted Jan 07, 2017. Accepted for publication Jan 12, 2017.

doi: $10.21037 /$ tcr.2017.02.27

View this article at: http://dx.doi.org/10.21037/tcr.2017.02.27

It's not necessary to stress out the bad outcome of patients with glioblastoma (GBM): after the classical Stupp's protocol (large surgical resection, radiotherapy and chemotherapy using temozolomide), the general survival rate is globally $9 \% 2$ years after diagnosis (1). This situation is mainly the consequence of a systematic tumor recurrence. Although not yet fully experimentally demonstrated, it is more and more accepted that these recurrences could be due to a subpopulation of GBM cells, the glioblastoma stem cells (GSCs) (2). At least, those GSCs have been demonstrated to play a role in GBM initiation and therapeutic resistance. Indeed, they are regarded as "stem cells" because they express stem cells markers, can differentiate into various cell types and are able to self-renew. Self-renewal is more restrictive than cell proliferation as it implies that at least one daughter cell is roughly identical to the mother cell, including the ability to perform the same number of cell cycles (3).

However, to self-renew, a normal somatic stem cell should be present in the niches, which could be functionally, rather than histologically, defined as a suitable cellular and molecular environment needed to maintain those stem cells quiescent most of the time, when development is done. Sometimes, those normal somatic cells undergo a cell cycle with an asymmetric division allowing them to give rise to a new stem cell and a progenitor cell. This last escapes the niche and actively undergoes cell cycles before differentiation (4). In the adult brain, including human, such niches hosting neural stem cells (NSC) have been so far located in the subventricular zones (SVZ) and in the dentatus gyrus (DG) of the hippocampus (5).

The cancer stem cells seem to be able to self-renew outside a normal somatic stem cells niche. In GBM in peculiar, although those GSCs exhibit a specific attraction to the SVZ $(6,7)$, some of them seem able to create their own niches outside the SVZ or the DG. Those "homemade" GSC niches are hypoxic and perivascular (8), with a higher capillary permeability as it is described in SVZ for the normal stem cells (9). However, as GBM is a highly invasive tumor, the question of how invading GSC maintain their stemness, including their self-renewal property, outside their homemade niches remains open and was addressed in a recent study (10).

Shingu et al. used an in vivo GBM model in which they invalidate three tumor suppressor genes expression specifically in adult NSC when located in the SVZ: Trp53, Pten and $Q k$. If Trp53 and Pten are known to be frequently invalidated by mutations in GBM (11), $Q k$ is less known (12). $Q k$ encodes QKI, a star-family RNA-binding protein involved in various aspects of RNA biology: RNA stability, RNA splicing, translation and miRNA processing. In GBM, it has been suspected to stimulate the differentiation of cancer cells (13).

First, Shingu et al. show that invalidation of $Q k$ in NSC is followed by a higher number of BrdU-positive cells and NSC cell number. But in vitro, it appears that 
the $Q k$ invalidation is responsible of a slow-down of the cell cycle with a higher degree of self-renewal in NSC, even in presence of low concentrations of both EGF and bFGF, the two classical growth factors for these cells. Meanwhile, both in vitro and in vivo, $Q k$ invalidation decreases the cell differentiation into neurons, astrocytes and oligodendrocytes.

Then, Shingu et al. compared the consequences of invalidation of Pten and $\operatorname{Tr} p 53$ with or without a $Q k$ invalidation in NSC. The invalidation of both Pten and Trp5 53 in NSC is followed by an enlargement of the SVZ due to a higher number of NSC but when those NSC leave the SVZ, they stop to self-renew and differentiate into neurons, astrocytes and oligodendrocytes. Moreover, in those $\mathrm{Pten}^{-/-}$and $\operatorname{Tr} 553^{-/-}$NSC mice, no glioma arise during the first 12 months of life. But when Shingu et al. look at the triple Pten ${ }^{-/-}, \operatorname{Trp} 53^{-/-}$and $\mathrm{Qk}^{-/-}$NSC mice, they could observe NSC outside the SVZ: in thalamus, in hypothalamus, in striatum or in the cortex, they found cells that, after undergoing the recombination, do not expressed differentiation markers but still express NSC markers (Ki67, Sox2). Once again, the absence of $Q k$ expression in NSC decreased the proliferation rate of NSC, even in absence of PTEN and $\mathrm{p} 53$ proteins, but increases the self-renewal ability in these cells with a lower dependence to growth factors in vitro.

But the breakthrough of this paper is the fact that $92 \%$ of mice invalidated for those three genes in NSC develop a glioma with a median survival time of 105 days. Nearly half of these gliomas arise in the cortex, the others from various parts in the central nervous system. Moreover, they could be classified as GBM as these tumors are invasive and exhibit necrosis and hypervascularity with neoangiogenesis. On the basis of transcriptomic profiling, those GBM represented all four types (proneural, neural, classical and mesenchymal).

The next question addressed by Shingu et al. concerns the role of QKI in NSC and how its absence can promote glioblastomagenesis. As QKI has been shown to regulate various aspects of RNA biology (12), they performed a transcriptomic and a proteomic comparison of NSC expressing or not $Q k$, with or without Pten and $\operatorname{Tr} p 53$ expression. Shingu et al. found 217 genes that were alternatively spliced in absence of QKI but also 290 upregulated and 343 down-regulated proteins. Then, they performed a photoactivable-ribonucleoside-enhanced crosslinking and immunoprecipitation (PAR-CLIP) in order to identify genes whose expression is directly modified by the QKI absence. By combining transcriptomic, proteomic and PAR-CLIP data, they identified 104 genes whose expression is changed, 73 genes that are alternatively spliced and 148 proteins whose level is modified in absence of QKI. The in-silico analysis of these modifications indicates that 15 molecular pathways are concerned by the absence of QKI and 12 of them are related to receptor signaling and trafficking. More precisely, about $40 \%$ of genes downregulated at the mRNA, at the protein level or at the splicing by the absence of QKI are involved in endocytosismediated receptor degradation including various components of endosomes and lysosomes.

This observation coupled to the fact that in absence of QKI, NSC self-renew in vitro at a low growth factors concentrations, raised the hypothesis that $Q k$ deletion maintains the self-renewal of stem cells outside the SVZ by down-regulating endolysosome-dependent degradation of growth factors receptors. In order to demonstrate this hypothesis, Shingu et al. demonstrate that in absence of QKI, there is a decrease of endolysosome levels in genetically modified-NSC but also in human GBM samples as various key components of this organelle appear to be down-regulated in TCGA data sets. Moreover, knockingdown Lamp1 (a key component of lysosome during autophagy) in NSC invalidated for Pten and Trp 53 allows the development of tumors. On the contrary, expression of Tfe 3 (a key component needed for endolysosome biogenesis) in NSC invalidated for Pten, Trp53 and $Q k$ decreases their self-renewal ability. The receptors and/or growth factors which are present at a higher levels in $Q k^{-/-}$NSC plasmatic membrane are Notch, Frizzled, Wnt5a and Wnt5b but also EGFR, a receptor whose gene is frequently amplified and/ or mutated in GBM (14).

This study is important by several aspects: (I) it describes a new in vivo experimental model of GBM; (II) it stress out the importance for a normal stem cell to be in a niche to be able to self-renew; (III) it also strengthens the possibility that GBM originate from NSC, as this point is still a matter of debate. However some points remain unsolved. Indeed, Shingu et al. emphasize that a decrease of endolysosome induced by the absence of QKI allows the NSC to be able to self-renew outside the SVZ, a situation that could pave the way to a GBM transformation. However, they do not demonstrate why a NSC outside the SVZ becomes a tumor cell or, in other words, why NSC able to self-renew in the SVZ do not undergo a tumor transformation most of the time. This study thus suggests that a cell (I) that is able to self-renew; but (II) that self-renews in the wrong place (outside the niche) will probably experience a tumor 
transformation. Therefore, if we do consider the role of the SVZ in self-renewal of NSC, we thus have also to take into account the role of SVZ in order to prevent their tumor transformation. This could be obtained in SVZ by keeping a relative quiescence of NSC after the development or by the modulation of symmetric or asymmetric cell division (15). Indeed, this process is clearly dependent about the orientation of the cleavage plane of the mother cell regarding to the presence of the ependymal wall in the SVZ. This orientation is obviously lost when NSC selfrenew outside the SVZ. The next questions are thus: how a stem cell, outside the niche but still able to self-renew, becomes a cancer stem cell? Is the loss of the cleavage plane ruling the asymmetrical or symmetrical division responsible of such a transformation?

\section{Acknowledgements}

None.

\section{Footnote}

Conflicts of Interest: The author has no conflicts of interest to declare.

\section{References}

1. Louis DN, Perry A, Reifenberger G, et al. The 2016 World Health Organization Classification of Tumors of the Central Nervous System: a summary. Acta Neuropathol 2016;131:803-20.

2. Lathia JD, Mack SC, Mulkearns-Hubert EE, et al. Cancer stem cells in glioblastoma. Genes Dev 2015;29:1203-17.

3. Wu J, Izpisua Belmonte JC. Stem Cells: A Renaissance in Human Biology Research. Cell 2016;165:1572-85.

4. Scadden DT. Nice neighborhood: emerging concepts of the stem cell niche. Cell 2014;157:41-50.

5. Silva-Vargas V, Crouch EE, Doetsch F. Adult neural stem

Cite this article as: Rogister B. Glioblastoma stem cells and the importance of endolysosomes to keep them in the niches. Transl Cancer Res 2017;6(Suppl 1):S87-S89. doi: 10.21037/ tcr.2017.02.27 cells and their niche: a dynamic duo during homeostasis, regeneration, and aging. Curr Opin Neurobiol 2013;23:935-42.

6. Kroonen J, Nassen J, Boulanger YG, et al. Human glioblastoma-initiating cells invade specifically the subventricular zones and olfactory bulbs of mice after striatal injection. Int J Cancer 2011;129:574-85.

7. Goffart N, Kroonen J, Di Valentin E, et al. Adult mouse subventricular zones stimulate glioblastoma stem cells specific invasion through CXCL12/CXCR4 signaling. Neuro Oncol 2015;17:81-94.

8. Hambardzumyan D, Bergers G. Glioblastoma: Defining Tumor Niches. Trends Cancer 2015;1:252-65.

9. Tavazoie M, Van der Veken L, Silva-Vargas V, et al. A specialized vascular niche for adult neural stem cells. Cell Stem Cell 2008;3:279-88.

10. Shingu T, Ho AL, Yuan L, et al. Qki deficiency maintains stemness of glioma stem cells in suboptimal environment by downregulating endolysosomal degradation. Nat Genet 2017;49:75-86.

11. Aldape K, Zadeh G, Mansouri S, et al. Glioblastoma: pathology, molecular mechanisms and markers. Acta Neuropathol 2015;129:829-48.

12. Darbelli L, Richard S. Emerging functions of the Quaking RNA-binding proteins and link to human diseases. Wiley Interdiscip Rev RNA 2016;7:399-412.

13. Hu J, Ho AL, Yuan L, et al. From the Cover: Neutralization of terminal differentiation in gliomagenesis. Proc Natl Acad Sci U S A 2013;110:14520-7.

14. Furnari FB, Cloughesy TF, Cavenee WK, et al. Heterogeneity of epidermal growth factor receptor signalling networks in glioblastoma. Nat Rev Cancer 2015;15:302-10.

15. Matsuzaki F, Shitamukai A. Cell Division Modes and Cleavage Planes of Neural Progenitors during Mammalian Cortical Development. Cold Spring Harb Perspect Biol 2015;7:a015719. 